\title{
Validation of methodology for assay, pharmaceutical equivalence, and comparative dissolution profile for tablets containing amlodipine besylate
}

Renata Micheli Martinez*, Jenifer Freitas da Silva, Larissa Regina Jorge, Rhye Lessa Ishikawa, Ana Paula Novelli, Talita Laiane Cardoso Cezar, Sandra Regina Georgetti, Marcela Maria Baracat, Rúbia Casagrande

Departament of Pharmaceutical Sciences, Londrina State University, University Hospital, 86039440 Londrina, Brazil.

\begin{tabular}{l}
\hline ARTICLE INFO \\
\hline Received on: 01/08/2019 \\
Accepted on: 24/09/2019 \\
Available online: 04/11/2019
\end{tabular}

Key words:

Amlodipine, generic, similar, drug products, quality control, validation.

\begin{abstract}
The amlodipine besylate is indicated as a first choice in the treatment of hypertension. Many similar and generic drug products companies were able to bring out to the market dosage forms containing amlodipine besylate with lower prices. In this context, the aim of this work was to validate a simple method for the determination of amlodipine content in tablets by ultraviolet spectrophotometry and to perform pharmaceutical equivalence and dissolution profile studies for three similar and one generic drug products and their respective innovator tablet containing amlodipine $(5 \mathrm{mg})$. The developed method for the determination of amlodipine content proved to be linear, precise, accurate, robust, and appropriate for employment in the quality control of tablets containing amlodipine besylate. The reference (R), similar (S1, S2, and S3), and generic (G) drug products all fulfilled the specifications for the tests of identification, average weight, hardness, friability, disintegration, drug content, content uniformity, and dissolution. However, in comparative dissolution profile studies, the dissolution efficiency of products G and S2 was statistically different from product R, which may indirectly lead to the unsuitable bioavailability and therapeutic inefficacy. Thus, there is a need for tighter legislation and inspection regarding the quality of pharmaceutical products already on the market.
\end{abstract}

\section{INTRODUCTION}

The amlodipine besylate is indicated as a drug of the first choice in the treatment of hypertension and angina pectoris due to myocardial ischemia. This drug relaxes the blood vessels, increasing the amount of blood and oxygen to the heart and reducing its workload. The high blood pressure makes the heart and the arteries a work overload that in the long term causes the heart and arteries to not function properly. This can cause damage to the blood vessels, resulting in brain-vascular diseases, heart attacks, and heart and renal failure. However, if blood pressure is controlled, for example, through the appropriate use of drug

"Corresponding Author

Renata Micheli Martinez, Departament of Pharmaceutical Sciences, Londrina State University, University Hospital, 86039440 Londrina, Brazil.E-mail: renatamimartinez@gmail.com products as tablets containing amlodipine besylate, these events can be avoided (Murdoch and Heel, 1991).

Many similar and generic drug products companies were able to bring out to the market dosage forms containing amlodipine besylate with lower prices, that are equivalent to those of reference when produced fulfilling the technical and legal requirements established, which can make them therapeutically interchangeable. In order to prove this, in vitro studies of pharmaceutical equivalence performing physical and physicochemical assays, and after in vivo studies of bioequivalence should be carried out (Brasil, 2010a). Differences in the results in vitro may lead to differences in bioavailability, impairing the bioequivalence between the products and, consequently, the effectiveness and safety of treatment when choosing a similar and generic drug product.

The physical and chemical characteristics of the drug, excipients, and the manufacturing technique employed in the production of the pharmaceutical form affect the dissolution of the drug and consequently its bioavailability and bioequivalence. This 
underscores the importance of the evaluation of the performance of the solid dosage forms, by performing in vitro tests, as dissolution profile studies, which allow understanding of how the drug is released as a function of time (de Brum et al., 2012, Santos et al., 2016).

In this context, in order to assure the manufacturing uniformity for the treatment of high blood pressure, as well as for quality control purposes, it is important to establish validated methods that can be performed as a simple and fast quantitation of the pharmaceutical dosage forms.

There are several methods described in the literature for amlodipine besylate dosage forms. Many methods are based on the high-performance liquid chromatography (HPLC) using UV; however, they require several pieces of expensive equipment/ reagents (Baranda et al., 2005; Klinkenberg et al., 2003; The United States Pharmacopeia, 2019). One spectrophotometry method was reported for assay of tablets containing amlodipine besylate (Feroz et al., 2014); however, the method used was not validated, and the solvent used was methanol, a chemical agent flammable and toxic, and whose residues-chemicals are difficult to deal with.

The ultraviolet spectrophotometry is widely used as an analytical tool in the drug product quality control. This technique is simple, fast, and selective without a need for sample pre-treatment or drug extraction (Rojas and Ojeda, 2009). In addition, these analysis methods are the lower cost and have a greater chance of application in compounding pharmacies and the majority of laboratory providers of routine quality control certificates. So, it is interesting to develop analytical method spectrophotometry safer and more environmentally friendly for the determination of amlodipine content, for example, by employing of $0.01 \mathrm{M} \mathrm{HCl}$ (mentioned on the American's Pharmacopeia for dissolution test of tablets containing amlodipine besylate) as drug diluent, hence, is a chemical residue, easy to neutralize or discharge.

Therefore, the aim of this work was to validate a simple and safer method for the determination of amlodipine content in pharmaceutical dosage forms by ultraviolet spectrophotometry, and to perform pharmaceutical equivalence and dissolution profile studies for three similar and one generic drug products and their respective innovator tablet containing amlodipine $(5 \mathrm{mg})$ from the local market of Londrina.

\section{MATERIALS AND METHODS}

\section{Drug products and chemicals}

The amlodipine besylate European Pharmacopoeia reference standard ( $99.9 \%$ of purity) was ceded by the Sandoz pharmaceutical industry (Cambé, Paraná, Brazil). The tablets containing amlodipine ( 5 or $6.95 \mathrm{mg}$ of amlodipine besylate) were purchased at pharmaceutical establishments (drugstores) from Londrina city, Paraná State, Brazil. Samples of the same batch for the reference $(R)$, generic $(G)$, and similar drug products named S1, S2, and S3 were analyzed. All samples used were within the shelf-life as stated by their manufacturers and as required by the current legislation (Brasil, 2010a). The similar drug products appear on the list of similar interchangeable of ANVISA.

Solvent hydrochloric acid was obtained from Biotec and Vetec (São Paulo, SP, Brazil). Ultra-pure water was produced by using a Milli-Q ${ }^{\circledR}$ system (Millipore ${ }^{\circledR}$, Bedford, MA, USA). All the chemicals used were of analytical grade.

\section{Equipment}

Ultrasonic bath Ultra Cleaner 1400 (Unique, Indaiatuba, SP, Brazil), UV/VIS spectrophotometer UV 1800 (Shimadzu ${ }^{\circledR}$, Corporation $^{\circledR}$, Kyoto, Japan); HPLC Shimadzu LC-10AT, detector SPD-10A UV/VIS (Shimadzu Corporation, Tokyo, Japan), dissolution test apparatus DT6 (Erweka ${ }^{\circledR}$, Heusenstamm, Germany); tablet friability apparatus TA-200 (Erweka ${ }^{\circledR}$, Heusenstamm, Germany); digital tablet hardness tester TBH 125 (ERWEKA ${ }^{\circledR}$, Heusenstamm, Germany); analytical balance Mettler 200 (Marshall $^{\circledR}$, New Hampshire, EUA) were used in this study. All equipment were properly qualified and calibrated previously to the analysis.

\section{Validation}

The validation process was performed according to the guidelines contained in resolution RE-166 of 2017 (MS-Anvisa, 2017). The absorbance of the solutions, dissolved with $0.01 \mathrm{M}$ $\mathrm{HCl}$, was determined at $239 \mathrm{~nm}$. Care has been taken with respect to the exposure of light, with the use of glass amber, and reading the solutions immediately, to prevent the degradation of amlodipine.

\section{Linearity}

It was determined by preparing standard solutions in the concentrations of $2.5,5.0,8.0,10,16,20$, and $25 \mu \mathrm{g} / \mathrm{ml}$ in triplicate. The linearity was evaluated by linear regression and linear correlation analysis.

\section{Precision}

The repeatability was evaluated on the same day for intra-day precision in six vessels independently. The evaluation of inter-day precision (intermediate precision) was performed on two different days by two analysts in six vessels independently. It was used standard solution amlodipine of $10 \mu \mathrm{g} / \mathrm{ml}$.

\section{Accuracy}

The method accuracy was reported as a recovery percentage of the known amount of standard solution (three concentration levels) added to the sample solution in a concentration of $5 \mu \mathrm{g} / \mathrm{ml}$. The concentration of the standard added-low, medium, and high was $2.0 ; 5.0$, and $8.0 \mu \mathrm{g} / \mathrm{ml}$ of amlodipine, respectively. Each solution was prepared in triplicate.

\section{Robustness}

Robustness of the method was determined for the preparation of a standard solution amlodipine of $10 \mu \mathrm{g} / \mathrm{ml}$ in which was evaluated the different manufacturers of the solvent hydrochloric acid, different $\mathrm{pHs}$, by the change of the wavelength $(238,239$, and $240 \mathrm{~nm}$ ), and by the stability of the solutions of 0 and 48 hours.

\section{Application of the method}

The methodology validated was tested with samples of the amlodipine besylate from tablets of the innovator drug product, and the results of the proposed method were compared with the results of the American's Pharmacopeia official method (The United States Pharmacopeia, 2019). 


\section{Pharmaceutical equivalence and comparative dissolution profile studies}

The assays for each sample/brand were performed according to the general methods of Brazilian Pharmacopeia (Brasil, 2010b), dissolution assay of the monograph for amlodipine besylate tablets as recommended at the American's Pharmacopeia (The United States Pharmacopeia, 2019), and according to the method for the determination of amlodipine content, validated in this work.

\section{Identification}

To identify the amlodipine in tablets, solutions of 10 $\mu \mathrm{g} / \mathrm{ml}$ from each sample and amlodipine reference standard were prepared as described in the determination of amlodipine content and separately scanned and overlapped in the range $\lambda 200-300 \mathrm{~nm}$.

\section{Average weight test}

Twenty randomly selected tablets were individually weighted by using an analytical balance. The average tablet weight and relative standard deviation (RSD) were calculated (Brasil, 2010b).

\section{Hardness test}

Ten randomly selected tablets were, separately, submitted to a radial force (Brasil, 2010b). The force in Newton (N) required to break them up was recorded and expressed as average \pm RSD.

\section{Friability test}

First, 20 randomly selected tablets were weighted and kept into a tablet friability apparatus to attain 100 rotations within 4 minutes. Thereafter, the tablets were gently cleaned up and reweighted. The friability (expressed as percentage of mass loss) was calculated according to equation (Brasil, 2010b):

Friability $=($ initial weight - final weight $) /$ initial weight $\times 100$.

\section{Disintegration test}

Disintegration test was performed using six randomly selected tablets and using $900 \mathrm{ml}$ water at $37^{\circ} \mathrm{C} \pm 1{ }^{\circ} \mathrm{C}$ as disintegration media (Brasil, 2010b).

\section{Determination of amlodipine content}

Twenty randomly selected tablets were weighted and powdered. A sample equivalent to $5 \mathrm{mg}$ of amlodipine was transferred to $100 \mathrm{ml}$ volumetric flask and dissolved with 80 $\mathrm{ml}$ of $0.01 \mathrm{M} \mathrm{HCl}$ by sonication for 10 minutes, followed by a mechanical shaker for 10 minutes. The volume was completed with the same solvent and it was homogenized and filtered. Five $\mathrm{ml}$ of this solution were placed into $25 \mathrm{ml}$ volumetric flask and the volume was filled up with $0.01 \mathrm{M} \mathrm{HCl}$. The absorbance of the solutions corresponding to samples and of amlodipine reference standard prepared in the same concentration was determined at $239 \mathrm{~nm}$, as validated previously in this work.

The experiments were carried out in triplicate, being the results expressed as percentage of declared quantity and by the average \pm RSD. Contents in percentage were calculated according to the equation:

$$
\text { Contents }=\left(A_{\mathrm{U}} \times C_{\mathrm{S}} \times W_{\mathrm{U}}\right) /\left(A_{\mathrm{S}} \times C_{\mathrm{U}} \times L\right) \times 100 .
$$

where $A_{\mathrm{U}}$ indicates the value of absorbance of the sample solution; $C_{\mathrm{S}}$ is the heavy mass exact of the reference standard, corresponding to amlodipine in $\mathrm{mg} ; W_{\mathrm{U}}$ is the average weight of the sample in $\mathrm{mg} ; A_{\mathrm{S}}$ is the absorbance of the standard solution; $C_{\mathrm{U}}$ is the mass exact of each socket of the test in $\mathrm{mg}$; and $L$ is the label claim (mg of amlodipine/tablet).

\section{Content uniformity}

Uniformity of unit dose was determined by the content uniformity method since the declared drug content is lower to 25 mg (Brasil, 2010b). Ten randomly selected tablets were exactly and individually weighted. The amount of amlodipine was reported in each unit deploying the same methodology for the determination of content and expressed in the individual results as a percentage of declared quantity. The Acceptance Value (AV) was calculated considering the case $1 \mathrm{a}(T \leq 101.5 ; 98.5 \leq X \leq 101.5 ; M=X)$ or case $1 \mathrm{~b}(T \leq 101.5 ; X>101.5 ; M=101.5)$ according to equations, respectively:

$$
\begin{gathered}
\mathrm{AV}=k \times s \\
\mathrm{AV}=[\mathrm{X}-101.5]+(k \times s)
\end{gathered}
$$

$T$ is the average value between the lower and the upper level of drug content requirement; $X$, the average individual content for each sample; $k$, the acceptability constant (2.4 for 10 tablets, and 2.0 for 20 tablets); $s$, the standard deviation of the individual content for each sample (Brasil, 2010b).

\section{Dissolution assay}

Six randomly selected tablets were individually placed into vessels of a dissolution apparatus fitted with a paddle (type-II apparatus) rotated at $75 \mathrm{rpm}$ for 30 minutes. The dissolution medium was $500 \mathrm{ml}$ of $0.01 \mathrm{M} \mathrm{HCl}$ at $37^{\circ} \mathrm{C} \pm 1^{\circ} \mathrm{C}$. Immediately after this time, $10 \mathrm{ml}$ samples were withdrawn from a zone midway between the surface of the dissolution medium and the top of the rotating paddle. The samples were filtered and absorbance was recorded at $239 \mathrm{~nm}$. The amounts of amlodipine besylate dissolved in the medium were calculated by comparing measurements for the reference standard at the same concentration prepared with $0.01 \mathrm{M} \mathrm{HCl}$. The percentage of the labeled amount of amlodipine dissolved $(Q)$ was calculated according to the equation:

$$
Q=\left(A_{\mathrm{U}} / A_{\mathrm{S}}\right) \times C_{\mathrm{S}} \times\left(M_{r 1} / M_{r 2}\right) \times V \times(1 / L) \times 100
$$

where $A_{\mathrm{U}}=$ absorbance of the sample solution; $A_{\mathrm{S}}=$ absorbance of the standard solution; $C_{\mathrm{S}}=$ concentration of the standard solution $(\mathrm{mg} / \mathrm{ml}) ; M_{r 1}=$ molecular weight of amlodipine, 408.88; $M_{r 2}=$ molecular weight of amlodipine besylate, 567.05; $V=$ volume of Medium, $500 \mathrm{ml} ; L=$ label claim (mg/Tablet) (The United States Pharmacopeia, 2019).

\section{Dissolution profiles}

The same set of operational conditions as for the dissolution assay was used. Twelve units of each sample were used in each dissolution profile (Brasil, 2010a). Samples were withdrawn at predefined time intervals $(2,5,10,15,20$, and 30 minutes $)$ and replaced with a fresh aliquot of dissolution medium, in order to maintain the volume of the medium. The samples were filtered and the amounts of amlodipine dissolved $(Q)$ at each time point were 
calculated by absorbance at $239 \mathrm{~nm}$ compared to the measurements for the reference standard at the same concentration, prepared with $0.01 \mathrm{M} \mathrm{HCl}$ (The United States Pharmacopeia, 2019).

In order to compare the dissolution profiles obtained, the drug dissolved amounts $(n=12)$ versus time were plotted, and the dissolution efficiency (DE) was determined. The DE was calculated from the area under the dissolution curve at time $t_{i}$ (measured using the trapezoidal rule) and expressed as a percentage of the area of the rectangle described by $100 \%$ dissolution at the same time (de Brum et al., 2012; Santos et al., 2016). Statistical treatments of DE results were based on variance analysis and $t$-test.

\section{Statistical analysis}

The average of the sample results was compared by paired Student's $T$-test, using the GraphPad Prism 7 package software (GraphPad Software Inc., San Diego, CA). The regression and linear correlation analysis were performed by the least-squared method, using the same software. Statistical significance was considered if $p \leq 0.05$.

\section{RESULTS AND DISCUSSION}

\section{Validation}

To perform pharmaceutical equivalence in vitro, it should be used official methods published in the Brazilian Pharmacopeia (Brasil, $2010 \mathrm{~b}$ ), or, if applicable, in other codes authorized by ANVISA (Brazil's National Health Surveillance Agency), or other applicable quality standards (Brasil, 2010a). However, there is no monograph that describes a method for the quality control of tablets containing amlodipine besylate in the Brazilian Pharmacopeia. In spite of reported spectrophotometry method for assay of tablets containing amlodipine besylate (Feroz et al., 2014), this method was not validated, and the solvent used was methanol, a chemical agent flammable and toxic, and whose residues-chemicals are difficult to deal with.

In this context, in order to apply the ultraviolet spectrophotometry method for drug quantitation that can be easily applied in the pharmaceutical laboratory routine due to simple and fast executions, we demonstrated the method's suitability for use to the determination of amlodipine content. The method was validated using the drug diluent $0.01 \mathrm{M} \mathrm{HCl}$, mentioned on the American's Pharmacopeia for dissolution test of tablets containing amlodipine besylate (The United States Pharmacopeia, 2019), hence, is a chemical residue, easy to neutralize or discharge and is less aggressive to the environment.

The results demonstrated that the correlation coefficient $(R)$ of the calibration curve was 0.999 , for all replicates $(n=3)$, according to RE 166/2017 (MS-Anvisa, 2017) minimum acceptable criterion (Fig. 1). Moreover, the method proved to be linear in a wide concentration range; consequently, it can be used for the determination of amlodipine content of other different doses of tablets available in the market. Given that results obtained from the six analytical solutions prepared, the method demonstrated repeatability with an $\mathrm{RSD} \%$ of up to $2.04 \%$ among samples prepared under the same conditions on the same day. The method also presented intermediate precision with RSD $\%$ of $4.07 \%$ among the results of six analytical solutions prepared by two different analysts on two different days, being within the maximum permitted of 5\% (MS-Anvisa, 2017).

The results for accuracy, at concentrations of $70 \%, 100 \%$, and $130 \%$, showed a mean recovery percentage of $100.82,98.40$,

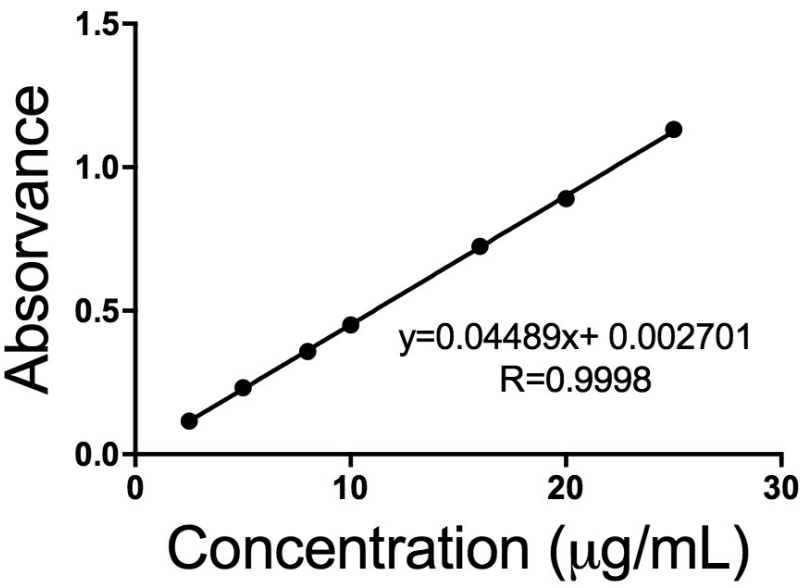

Figure 1. Calibration curve of amlodipine through absorption spectrophotometry in the UV region at $239 \mathrm{~nm}$.

and 99.55 , respectively. Mean recoveries are within the $98 \%$ to $102 \%$ range, which is in agreement with the literature (Green, 1996). The estimated limit of detection and limit of quantitation (MS-Anvisa, 2017) of the method were 0.18 and $0.59 \mu \mathrm{g} / \mathrm{ml}$, respectively. The method was shown to be robust since the results of amlodipine content were not significantly different for changes within the manufacturers of the solvent hydrochloric acid, Biotec and Vetec $(p=0.26)$, $\mathrm{pHs}$ of 1.92 and $1.12(p=0.99)$, by the change of the wavelength of 238 and $239 \mathrm{~nm}(p=0.26)$, and of 239 and $240 \mathrm{~nm}(p=0.24)$, and by the stability of the solutions of 0 and 48 hours $(p=0.17)$.

Moreover, the results showed that there were no significant differences between the validated proposed method (average of content $=102.27 \%$ ) and the American's Pharmacopeia official method (average of content $=101.82 \%, p=0.61$ ) for amlodipine besylate from tablets of the reference drug product. Accordingly, the developed method for the determination of amlodipine content proved to be linear, precise, accurate, robust, and appropriate technique that can be employed in the quality control of tablets containing amlodipine besylate.

\section{Pharmaceutical equivalence and comparative dissolution profile studies}

Similar drug products had to undergo the same tests as generic drug products and must be bioequivalent to its innovator drug product. However, despite the current rigid legislation regarding good manufacturing practice and quality control, it is still possible to find drug products that deviate from quality standards being marketed in Brazil (Giordani and de Melo, 2012; Knappmann and de Melo, 2010; Locatelli et al., 2015; Marques and Andrade et al., 2018; Pinho and Storpirtis, 2016).

Herein, the active substance in all the samples analyzed was identified as amlodipine besylate since their absorption spectra were overlapped with the spectrum of the reference standard, with the same maximum absorption (Fig. 2). All analyzed samples G, S1, S2, and S3 were pharmaceutical equivalents to the R, once they fulfilled all the in vitro quality requirements. The results of the average weight, hardness, friability, disintegration, drug content, content uniformity, and dissolution are demonstrated in Tables 1 and 2. Although all samples comply with the specifications for these quality attributes, it 
was observed statistically significant differences between the $\mathrm{R}$ and G, S1, S2, and S3 in the average weight and hardness parameters, and between the $\mathrm{R}$ and $\mathrm{S} 1$ and $\mathrm{S} 3$ in the disintegration parameter (Table 1).

Considering the average weight test, the lower average value obtained was $62.24 \mathrm{mg}$ (S3), whereas the greater was 202.57 $\mathrm{mg}(\mathrm{R})$ (Table 1). Hence, the variation limit established was \pm $10 \%$ for $\mathrm{S} 3$ and $\pm 7.5 \%$ for $\mathrm{R}, \mathrm{G}, \mathrm{S} 1$, and $\mathrm{S} 2$ since the Brazilian Pharmacopoeia recommends (Brasil, 2010b): average weight $\leq 80 \mathrm{mg}, \pm 10 \%$ variation; $80 \mathrm{mg} \leq$ average weight $\leq 250 \mathrm{mg}, \pm$ $7.5 \%$ variation; and $\geq 250 \mathrm{mg}, \pm 5 \%$ variation are acceptable. For tablets, only two unities may not reach such specification interval, and all unities must not have weight lower or upper the double of the specified limits. Thus, all samples evaluated varied within the specified intervals: $187.37 \mathrm{mg} \leq \mathrm{R} \leq 217.76 \mathrm{mg} ; 179.55 \mathrm{mg} \leq \mathrm{G} \leq$ $208.66 \mathrm{mg} ; 167.82 \mathrm{mg} \leq \mathrm{S} 1 \leq 195.04 \mathrm{mg} ; 149.28 \mathrm{mg} \leq \mathrm{S} 2 \leq 173.49$ $\mathrm{mg}$; and $68.46 \mathrm{mg} \leq \mathrm{S} 3 \leq 56.01 \mathrm{mg}$.

Tablet hardness was in the recommended range of more than 30 Newton (de Brum et al., 2012, Giordani and de Melo, 2012), the lower hardness value obtained was $38.6 \mathrm{~N}$ (S3), and the greater was $107.3 \mathrm{~N}$ (R) (Table 1). In spite of the hardness test to be informative (Brasil, 2010b), this is an important

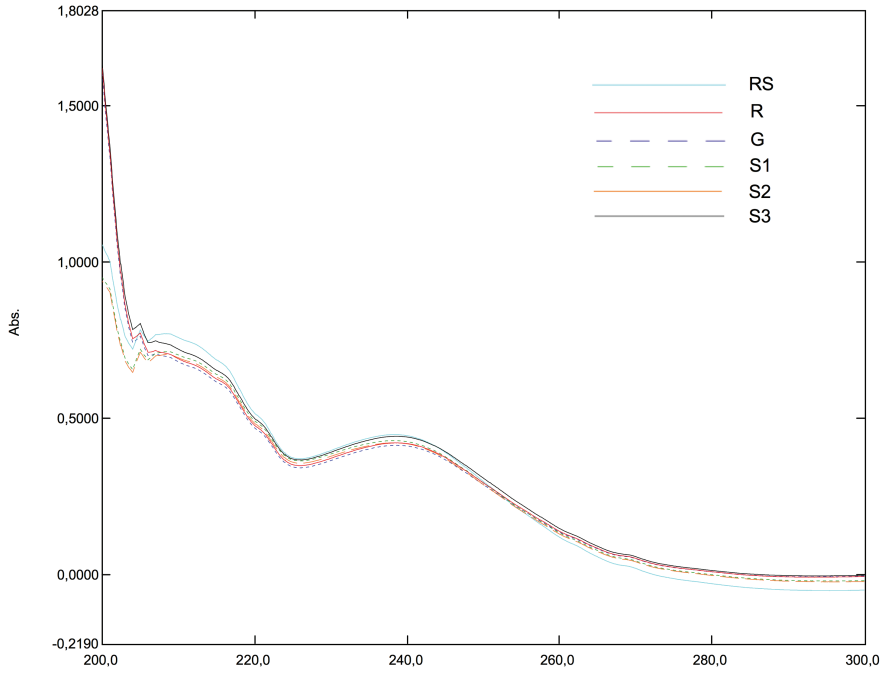

Figure 2. UV spectra for amlodipine reference standard (RS), and tablets of reference, generic, and three similar drug products equivalent to $10 \mu \mathrm{g} / \mathrm{ml}$ of amlodipine in $\mathrm{HCl} 0.01 \mathrm{M}$. R, reference product; $\mathrm{G}$, generic product; $\mathrm{S} 1-3$ similar products; wavelength range $\lambda 200-400 \mathrm{~nm}$.

Table 1. Average weight, hardness, friability, and disintegration for the tablets containing amlodipine $(5 \mathrm{mg})$.

\begin{tabular}{ccccc}
\hline Sample & $\begin{array}{c}\text { Weight } \\
(\mathbf{m g}) \pm \mathbf{R S D}\end{array}$ & $\begin{array}{c}\text { Hardness } \\
(\boldsymbol{N}) \pm \mathbf{R S D}\end{array}$ & Friability (\%) & Disintegration (sec) \\
\hline $\mathrm{R}$ & $202.57 \pm 0.87$ & $107.3 \pm 7.98$ & 0.08 & $3.42 \pm 1.22$ \\
$\mathrm{G}$ & $194.11 \pm 0.84^{*}$ & $47.0 \pm 7.09^{*}$ & 0.20 & $3.50 \pm 1.52$ \\
$\mathrm{~S} 1$ & $181.43 \pm 1.29^{*}$ & $66.0 \pm 5.50^{*}$ & 0.27 & $4.37 \pm 2.66^{*}$ \\
$\mathrm{~S} 2$ & $161.38 \pm 1.93^{*}$ & $59.7 \pm 13.21^{*}$ & 0.07 & $3.62 \pm 9.63$ \\
$\mathrm{~S} 3$ & $62.24 \pm 1.38^{*}$ & $38.6 \pm 5.70^{*}$ & 0.35 & $4.43 \pm 2.77^{*}$ \\
\hline
\end{tabular}

$\mathrm{R}=$ reference product, $\mathrm{G}=$ generic product, and $\mathrm{S} 1-3=$ similar products. $\mathrm{RSD}=$ relative standard deviation $(\%), \mathrm{N}=$ Newton

${ }^{*} p<0.0001$, statistically significant difference as compared to the reference drug product.
Table 2. Results of the drug content, content uniformity, and dissolution test for the tablets containing amlodipine $(5 \mathrm{mg})$.

\begin{tabular}{cccc}
\hline Sample & $\begin{array}{c}\text { Drug content } \\
(\%) \pm \text { RSD }\end{array}$ & AV & Average dissolved (\%) \pm RSD \\
\hline R & $102.27 \pm 1.36$ & 12.06 & $100.92 \pm 1.28$ \\
G & $99.59 \pm 0.54$ & 7.44 & $102.62 \pm 3.41$ \\
S1 & $102.31 \pm 1.07$ & 15.57 and $9.25^{*}$ & $95.18 \pm 2.71$ \\
S2 & $103.59 \pm 4.45$ & 10.58 & $104.97 \pm 3.10$ \\
S3 & $106.52 \pm 0.39$ & 8.33 & $101.04 \pm 1.49$ \\
\hline
\end{tabular}

$\mathrm{R}=$ reference product, $\mathrm{G}=$ generic product, and $\mathrm{S} 1-3=$ similar products.

$\mathrm{RSD}=$ relative standard deviation $(\%), \mathrm{AV}=$ acceptance value, from the content uniformity assay.

*AV for the second stage with more 20 units.

feature in the definition of the variables of the productive process such as choice of excipients and appropriate concentrations, and production technology. Tablets are subject to mechanical shocks during production, packing, transportation, and distribution. For this reason, they should possess a suitable mechanical resistance (Giordani and de Melo, 2012).

The tests of hardness and friability evaluate the mechanical resistance of the tablets, which ensures the physical integrity of the drug product in the act of dispensation (Ghorab et al., 2004). The friability test resulted in a small loss of mass, well below the limit recommended of $<1.5 \%$ (Brasil, 2010b), for all five marketed brands of amlodipine besylate tablets, with the lower value obtained of $0.08 \%(\mathrm{R})$, and greater of $0.35 \%(\mathrm{~S} 3)$ (Table 1). High friability means that the drug product is more likely to suffer mechanical erosion, which may trigger loss of the active principle and thus compromise its efficacy.

Disintegration test is related to the capacity of solid pharmaceutical forms to release their active principles, because before their solubilization, the tablets must disintegrate into small particles, increasing the contact surface with the dissolution medium and favoring absorption and bioavailability of the drug (Giordani and de Melo, 2012). All samples proved satisfactory, as all tablets had completely disintegrated within well less than 30 minutes (Table 1).

It is interesting to note, the tablets with lower average weight (S3) also showed a lower hardness and higher friability, in this sense, the tablets with higher weight average (R) also showed higher hardness and lower friability. Figure 3A shows a positive correlation $(\mathrm{R}=0.625)$ obtained between the average weight and the hardness of the tablets. Therefore, the greater the average weight, the greater is the tablet's hardness. This trend may be explained by the increase in the space occupied and by increase in the relative contribution of diluents that may result in increased interactions between the powder particles, leading to higher hardness for tablets. Moreover, a negative correlation $(R$ $=-0.646)$ was observed between the hardness and the friability (Fig. 3B).

The average amlodipine contents in the samples varied between $99.59 \%(\mathrm{G})$ and $106.52 \%(\mathrm{~S} 3)$ (Table 2), within the pharmacopeia specification $(90 \%-110 \%)$ (The United States Pharmacopeia, 2019). The RSD values obtained for such experiments were greater to S2 (4.45), corroborated by the results of greater RSD in the average weight (1.93), hardness (13.21), and 

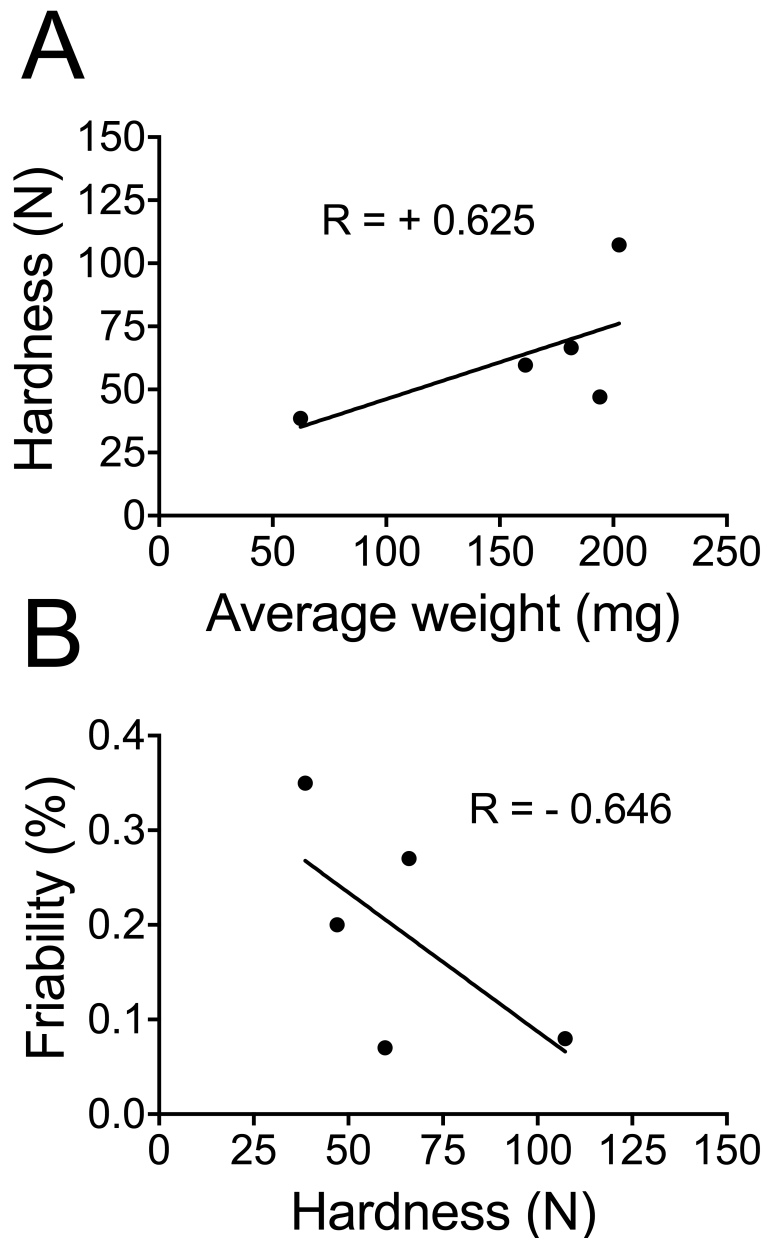

Figure 3. Correlation between average weight and hardness, and between friability and hardness of the tablets containing amlodipine besylate. $R^{2}$, determination coefficient; N, Newton. (A) Positive signal $(+)$ indicates a directly proportional correlation. (B) Negative signal (-) indicates an inversely proportional correlation.

in the disintegration (9.63), for this marketed brand, that reinforces the larger change of the results.

For the content uniformity, the calculated AV of the first 10 dosage units must be less than 15 . Herein, the samples R, G, $\mathrm{S} 2$, and $\mathrm{S} 3$ were suitable in the acceptable value range, 12.06, $7.44,10.58$, and 8.33, respectively (Table 2). On the other hand, $\mathrm{S} 1$ presented AV more than 15 (15.57) for the first 10 units. Thus, was tested the next 20 units, calculated the AV, and the sample S1 varied within the pharmacopeia specification, being also approved, since the final AV of the 30 dosage units was less than 15 (9.25), and no individual content of any dosage units was less or more than specified (Brasil, 2010b).

According to the American's Pharmacopeia (The United States Pharmacopeia, 2019), at least 75\% (Q) of amlodipine must be dissolved into the dissolution medium within 30 minutes. As presented in Table 2, all samples were approved at the first test stage. Differences in the characteristics of both active principle and excipients, such as routes of obtainment, as well as the productive processes by the different similar and generic drug products companies may affect the in vitro quality, bioequivalence, and therapeutically interchangeable of these drug products with the innovator drug product. For example, variations in the tableting force and velocity can influence the organization degree and consolidation state of solid particulate materials, and consequently impact the dissolution time of tablets. Thus, it is necessary to equilibrate the physical (average weight, hardness, friability, and disintegration), physicochemical (drug content, content uniformity, and dissolution profile), and biopharmaceutical (and bioavailability) properties of the drug products aiming its efficacy, safety, and quality assurance (Kumar et al., 2016).

Comparative dissolution profile studies can be used to determine the speed of availability and amount of the active principle available, relative to its innovator drug product. This is an important assay for determining the pharmaceutical equivalence between drug products and it must be carried out before performing bioequivalence of amlodipine besylate tablets (Brasil, 2010a). Moreover, the in vitro dissolution profiles are the most sensitive and trustworthy predictive methods for the in vivo active principle availability, or bioavailability (Anderson et al., 1998). Dissolution profile studies are a valuable tool in the batch-by-batch quality control, and for establishing similarity between drug products from different brands; however, is not an obligatory assay in routine quality control (Marques de Andrade et al., 2018).

According to American's Pharmacopeia (The United States Pharmacopeia, 2019), the dissolution test may be carried out by collecting only one aliquot after 30 minutes, in which $75 \%$ of the amlodipine must be dissolved in the dissolution medium after this interval. Table 2 shows that all the drug products would be approved if only this one point was used in the analysis. However, studies have shown that drug products are approved in the general quality tests, but many are disapproved in the dissolution profile (Giordani and de Melo, 2012; Marques de Andrade et al., 2018).

vure 4 shows the results of the dissolution profiles of the five marketed brands of amlodipine besylate tablets ( $R, G$, $\mathrm{S} 1, \mathrm{~S} 2$, and S3). It is noteworthy that the maximum average of amlodipine dissolved percent was reached within 5 minutes for the drug product $\mathrm{S} 1$, and in 10 minutes for drug products $\mathrm{R}, \mathrm{G}$, $\mathrm{S} 2$, and S3. Since all the drug products showed more than $85 \%$ of the active principle dissolved within 15 minutes, the similarity factor $\mathrm{f} 2$ and difference factor $\mathrm{fl}$ lose its power of discrimination among the profiles and thus were not calculated (Brasil, 2010a). Amlodipine besylate tablets available in the Pakistani market also were very rapidly dissolving, though in other dissolution mediums (Feroz et al., 2014). The RSD for the first points of collection may not exceed $20 \%$, and for the other points considered the maximum of $10 \%$ (Brasil, 2010a). The results of the dissolution profiles demonstrated that in the time of 2 minutes, all drug products showed RSD lower than $20 \%$, and in other times, $(5,10,15,20$, and 30 minutes) R, S1, S2, and S3 showed RSD lower than $10 \%$, except the product $\mathrm{G}$ which presented a RSD of $10.3 \%$ at the time of 30 minutes.

A statistical comparison between two dissolution profiles has the purpose of testing the possibility of interchange ability between the reference, and a similar or generic drug product (Anderson et al., 1998). The results in Figure 4B show that drug products $\mathrm{R}, \mathrm{S} 1$, and $\mathrm{S} 3$ presented dissolution efficiency very close one from each other. On the other hand, the dissolution efficiency 

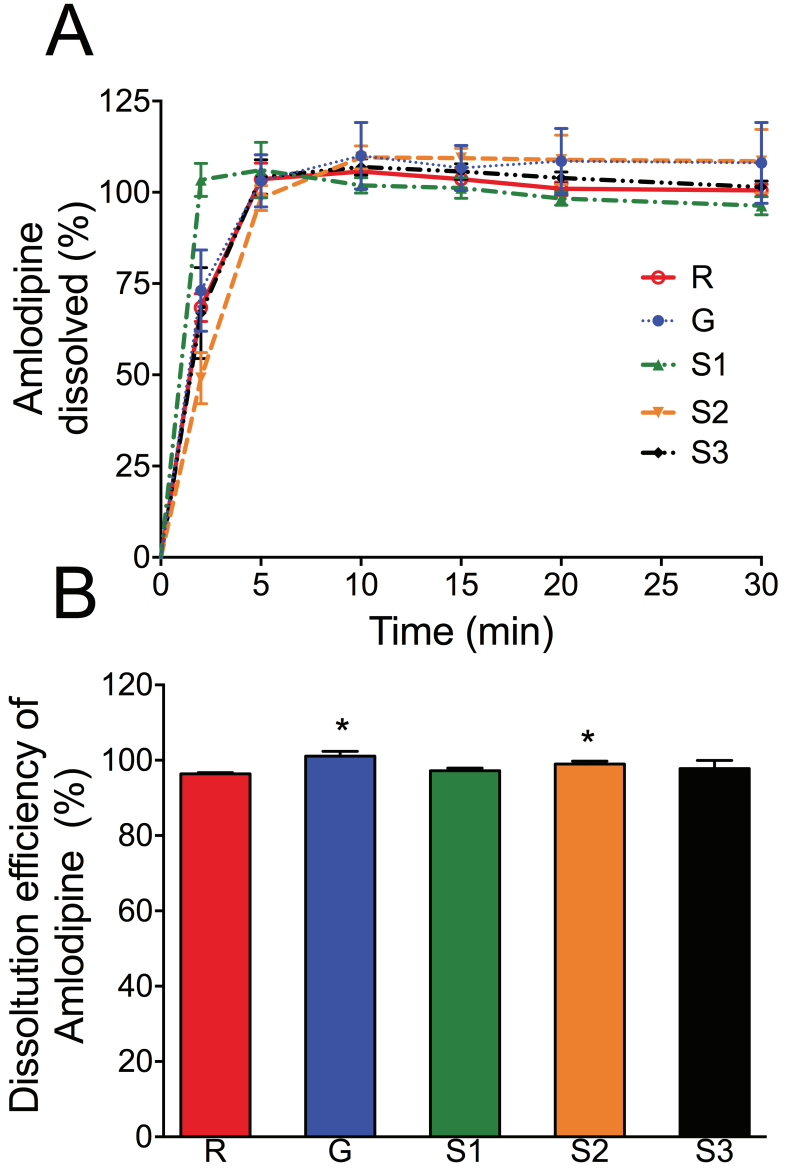

Figure 4. Dissolution profiles and dissolution efficiency of amlodipine besylate from tablets of reference, generic, and three similar drug products $(5 \mathrm{mg})$. R, reference product; G, generic product; S1-3 similar products; paddle (type-II apparatus); $75 \mathrm{rpm} ; 2,5,10,15,20$, and 30 minutes; $500 \mathrm{ml} ; 0.01 \mathrm{M} \mathrm{HCl} ; 37^{\circ} \mathrm{C}$ $\pm 1{ }^{\circ} \mathrm{C} ; n=12 .{ }^{*} p<0.004$ compared to the reference product.

of products $\mathrm{G}$ and $\mathrm{S} 2$ was statistically different from product $\mathrm{R}$ ( $p<0.001$ and 0.004 , respectively).

Dissolution efficiency is a useful measurement of batch homogeneity with respect to dissolution and this too can be used to monitor the homogeneity of batches (Anderson et al., 1998). Inadequate drug dissolution greatly compromises the required effect on the organism, resulting in poor therapeutic efficacy, and may represent a risk to a patient's health. Corroborating the results of dissolution profiles, drug products $\mathrm{G}$ and $\mathrm{S} 2$ also presented high RSD on the test of dissolution, and even S2 presented greater RSD on average weight, hardness, disintegration, and drug content tests. In addition, it has already been shown that capsule and tablet containing amlodipine failed the tests of content uniformity and hardness, respectively (Malesuik et al., 2006).

According to the Biopharmaceutics Classification System, amlodipine besylate is a class III drug, with high solubility and low permeability, which means that permeation is the rate-limiting step for drug absorption (Papich and Martinez, 2015). In this way, even if the drug products have demonstrated dissolution efficiency similar, it is not possible to assert that they are therapeutically equivalent to the R, which only must be confirmed by performing bioequivalence studies.

It was not observed that correlation between the hardness and the dissolution times, thus, for the studied samples, the tablet drug dissolution may be more dependent on the qualitative aspects of the formulations, for example, the quantity of diluent, than on the processing parameters, for example, compression force. Only the product S1 has in its composition, the excipient lactose monohydrate, and was the one that released more quickly of the active ingredient, with the maximum average of amlodipine dissolved percent reached within 5 minutes. The presence of diluents, very hydrophilic such as lactose, may enhance the capture of fluid, and consequently, increase the wettability of the particles accelerating the speed of release of drugs (Rowe et al., 2012). Moreover, the diluent used in the different products evaluated included the microcrystalline cellulose; however, only the S1 product does not have in its composition the diluent calcium phosphate dibasic.

It is important to mention that to have appropriate similar and generic product selection, healthcare professionals require ample information on interchangeability for substitution of products. Moreover, there is a need for tighter legislation and inspection regarding the quality of similar and generic drug products already on the market, which when implemented will further enhance the quality of drug products available to the Brazilian population, besides increasing the competitiveness of Brazilian manufacturers of similar and generic drug products.

\section{CONCLUSION}

The simple, fast, safer, and low-cost validated method, by ultraviolet spectrophotometry, is an excellent alternative to sophisticated instrumental methods and can be easily applied in the quality control routine of tablets containing amlodipine besylate.

The reference $(\mathrm{R})$, similar (S1, S2, and S3), and generic (G) drug products all fulfilled the pharmacopeia specifications for the tests of average weight, hardness, friability, disintegration, drug content, content uniformity, and dissolution. However, in comparative dissolution profile studies, the dissolution efficiency of drug products $\mathrm{G}$ and $\mathrm{S} 2$ were statistically different from product $\mathrm{R}$, which may indirectly lead to the unsuitable bioavailability of the active principle and therapeutic inefficacy. Nevertheless, the therapeutically interchangeable must be confirmed by performing bioequivalence studies.

\section{ACKNOWLEDGMENTS}

The authors greatly appreciate to the Laboratory of Pharmaceutical Analysis of the State University of Londrina for providing the technical support involved in the analysis, and Sandoz pharmaceutical industry (Cambé, Paraná, Brazil) for the donation of the reference standard.

\section{CONFLICT OF INTEREST}

The authors declared that they have no conflict of interest.

\section{REFERENCES}

Anderson NH, Bauer M, Boussac N, Khan-Malek R, Munden P, Sardaro M. An evaluation of fit factors and dissolution efficiency for the comparison of in vitro dissolution profiles. J Pharm Biomed Anal, 1998; 17(4-5):811-22. 
Baranda AB, Etxebarria N, Jiménez RM, Alonso RM. Development of a liquid-liquid extraction procedure for five 1,4-dihydropyridines calcium channel antagonists from human plasma using experimental design. Talanta, 2005; 67(5):933-41.

Brasil. Resolução RDC No. 31, de 11 de agosto de 2010. Agência Nac. Vigilância Sanitária, pp 36-8, 2010a.

Brasil M das SAN de VS. Farmacopéia Brasileira. Diário Of. da União, 2010b.

de Brum TF, Laporta L V, Pons Júnior FR, Gonçalves CA, dos Santos MR. Pharmaceutical equivalence and comparative study of dissolution profiles of generic drugs containing acetaminophen. Rev Ciencias Farm Basica e Apl, 2012; 33(3):373-8.

Feroz M, Razvi N, Ghayas S, Anjum F, Ghazal L, Siddiqui SA. Assessment of pharmaceutical quality control and equivalence of various brands of amlodipine besylate $(5 \mathrm{mg})$ tablets available in the pakistani market under biowaiver conditions. Int J Pharm Pharm Sci, 2014; 6(2): 909-13.

Ghorab MM, Abdel-Salam HM, El-Sayad M a, Mekhel MM Tablet formulation containing meloxicam and beta-cyclodextrin: mechanical characterization and bioavailability evaluation. AAPS PharmSciTech, 2004; 5(4):59.

Giordani MA, de Melo EB. Comparative study of the pharmacopeial quality and dissolution profiles of generic and other drug forms of sodium metamizole (dipyrone) sold in Brazil. Rev Ciencias Farm Basica e Apl, 2012; 33(3).

Green JM. Peer reviewed: a practical guide to analytical method validation. Anal Chem, 1996; 68(9):305A-9A.

Klinkenberg R, Streel B, Ceccato A. Development and validation of a liquid chromatographic method for the determination of amlodipine residues on manufacturing equipment surfaces. J Pharm Biomed Anal, 2003; 32(2):345-52.

Knappmann AL, de Melo EB. [Quality of over-the-counter medicines: a study with dipyrone brands commercialized in a drugstore in Cascavel city (Paraná, Brazil)]. Cien Saude Colet, 2010; 15 Suppl 3: 3467-76.

Kumar D, Singh J, Antil M, Kumar V. Quality control of tablets: a review. Int J Univers Pharm Bio Sci, 2016; 5(4):53-67.

Locatelli T, da Silva AK, Sausen TR. Pharmaceutical equivalence of anti-hypertensive tablets distributed by a Brazilian governmental program. Lat Am J Pharm, 2015; 34(8):1601-6.

Malesuik MD, Cardoso SG, Lanzanova FA, Bajerski L, Dorigoni E. Development of dissolution test and comparative study of tablets and compounded capsules containing amlodipine. Rev Ciencias Farm Basica e Apl, 2006; 27(1):37-49.

Marques de Andrade G, Carvalho Ribeiro C, Castro Alves Plank B, Oliveira do Couto R. Pharmaceutical equivalence and comparative dissolution profile studies for coated tablets containing Verapamil hydrochloride. J Appl Pharm Sci, 2018; 5 (1): 57-71.

MS-Anvisa. RDC No 166, de 24 de julho de 2017. Diário Of da República Fed do Bras, 2017; 2017:22.

Murdoch D, Heel RC. Amlodipine: a review of its pharmacodynamic and pharmacokinetic properties, and therapeutic use in cardiovascular disease. Drugs, 1991; 41(3):478-505.

Papich MG, Martinez MN. Applying biopharmaceutical classification system (BCS) criteria to predict oral absorption of drugs in dogs: challenges and pitfalls. AAPS J, 2015; 17(4):948-64.

Pinho J de JRG de, Storpirtis S. In vitro comparative study of biopharmaceutics properties of metformin hydrochloride tablets marketed in Brazil. RBCF, Rev Bras Cienc Farm, 2016; 37(1):95-105.

Rojas FS, Ojeda CB. Recent development in derivative ultraviolet/visible absorption spectrophotometry: 2004-2008: a review. Anal Chim Acta, 2009; 635:22-44.

Rowe, Raymond C, Sheskey PJFME. Handbook of pharmaceutical excipients: pharmaceutical excipients. Am Pharm Assoc, 2012; 6: 1-855.

Santos BWL, Caldas ED, Da Silva MV. Nimesulide: dissolution profile, validation of analytical methods for capsules, and assessment of product quality. Int J Pharm Pharm Sci, 2016; 8(1):27-32.

The United States Pharmacopeia. 41th edition, Pharmacopeial Convention, Rockville, MD, 2019.

How to cite this article:

Martinez RM, da Silva JF, Jorge LR, Ishikawa RL, Novelli AP, Cezar TLC, Georgetti SR, Baracat MM, Casagrande RB. Validation of methodology for assay, pharmaceutical equivalence, and comparative dissolution profile for tablets containing amlodipine besylate. J Appl Pharm Sci, 2019; 9(11):093-100. 